\title{
Molecular Engineering of Curcumin, an Active Constituent of Curcuma longa L. (Turmeric) of the Family Zingiberaceae with Improved Antiproliferative Activity
}

\author{
Amena Ali ${ }^{1, *,+(\mathbb{D}}$, Abuzer Ali ${ }^{2,+}$, Abu Tahir ${ }^{3}$, Md. Afroz Bakht ${ }^{4}\left(\mathbb{D}\right.$, Salahuddin ${ }^{5}$ and Mohamed Jawed Ahsan ${ }^{6, *(\mathbb{D})}$ \\ 1 Department of Pharmaceutical Chemistry, College of Pharmacy, Taif University, P.O. Box 11099, \\ Taif 21944, Saudi Arabia \\ 2 Department of Pharmacognosy, College of Pharmacy, Taif University, P.O. Box 11099, Taif 21944, Saudi Arabia; \\ abuali@tu.edu.sa \\ 3 Department of Pharmacology, Raghukul College of Pharmacy, Bhopal 462 003, India; \\ aliabutahir2009@gmail.com \\ 4 Department of Chemistry, College of Science and Humanity Studies, Prince Sattam Bin Abdulaziz University, \\ P.O. Box 83, Al-Kharj 11942, Saudi Arabia; bakhtpharm@gmail.com \\ 5 Department of Chemistry, Noida Institute of Technology (Pharmacy Institute), Knowledge Park-2, \\ Greater Noida 201 306, India; sallu_05@yahoo.co.in \\ 6 Department of Pharmaceutical Chemistry, Maharishi Arvind College of Pharmacy, Ambabari Circle, \\ Jaipur 302 039, India \\ * Correspondence: amrathore@tu.edu.sa (A.A.); jawedpharma@gmail.com (M.J.A.) \\ + Authors contributed equally.
}

Citation: Ali, A.; Ali, A.; Tahir, A.; Bakht, M.A.; Salahuddin; Ahsan, M.J. Molecular Engineering of Curcumin, an Active Constituent of Curcuma longa L. (Turmeric) of the Family Zingiberaceae with Improved Antiproliferative Activity. Plants 2021, 10, 1559. https://doi.org/10.3390/ plants10081559

Academic Editor: Octavian Tudorel Olaru

Received: 20 June 2021

Accepted: 26 July 2021

Published: 29 July 2021

Publisher's Note: MDPI stays neutral with regard to jurisdictional claims in published maps and institutional affiliations.

Copyright: (c) 2021 by the authors. Licensee MDPI, Basel, Switzerland. This article is an open access article distributed under the terms and conditions of the Creative Commons Attribution (CC BY) license (https:/ / creativecommons.org/licenses/by/ $4.0 /)$.

\begin{abstract}
Cancer is the world's second leading cause of death, accounting for nearly 10 million deaths and 19.3 million new cases in 2020. Curcumin analogs are gaining popularity as anticancer agents currently. We reported herein the isolation, molecular engineering, molecular docking, antiproliferative, and anti-epidermal growth factor receptor (anti-EGFR) activities of curcumin analogs. Three curcumin analogs were prepared and docked against the epidermal growth factor receptor (EGFR), revealing efficient binding. Antiproliferative activity against $60 \mathrm{NCI}$ cancer cell lines was assessed using National Cancer Institute (NCI US) protocols. The compound $\mathbf{3 b}, \mathbf{c}$ demonstrated promising antiproliferative activity in single dose (at $10 \mu \mathrm{M})$ as well as five dose $(0.01,0.10,1.00$, 10 , and $100 \mu \mathrm{M}$ ). Compound 3c inhibited leukemia cancer panel better than other cancer panels with growth inhibition of $50 \%\left(\mathrm{GI}_{50}\right)$ values ranging from 1.48 to $2.73 \mu \mathrm{M}$, and the most promising inhibition with $\mathrm{GI}_{50}$ of $1.25 \mu \mathrm{M}$ was observed against leukemia cell line SR, while the least inhibition was found against non-small lung cancer cell line NCI-H226 with GI $_{50}$ value of $7.29 \mu \mathrm{M}$. Compounds $3 \mathbf{b}, \mathbf{c}$ demonstrated superior antiproliferative activity than curcumin and gefitinib. In molecular docking, compound $3 \mathrm{c}$ had the most significant interaction with four $\mathrm{H}$-bonds and three $\pi-\pi$ stacking, and compound $3 \mathrm{c}$ was found to moderately inhibit EGFR. The curcumin analogs discovered in this study have the potential to accelerate the anticancer drug discovery program.
\end{abstract}

Keywords: antiproliferative agents; anti-EGFR; curcumin analogues; molecular docking; cancer cell lines

\section{Introduction}

Natural products (NPs) have been used to treat human ailments since the dawn of medicine. NPs have historically been a highly prolific source of new medicines since the time immemorial in most of the civilizations. NPs continue to offer a diverse set of structural templates for drug discovery and development, which was heightened in the 1970s and 1980s [1,2]. Many of today's small molecule therapeutics can be traced back to NPs, which are anticipated to have provided or inspired the development of $50-70 \%$ of all agents currently in clinical use [3]. Studies focused on the isolation, structural elucidation, and biological activities of substances derived from NPs began around the end 
of the 18th century and introduction of column chromatography revolutionized an era of NP chemistry [4]. A number of anticancer drugs have been developed by chemically modifying plant NPs. Epothilone B, derived from Sorangiumcellulosum, has been chemically modified to Ixabepilone [5]. Camptothecin, an alkaloid derived from Camptotheca acuminate, has been modified into various anticancer agents including Karenitecin (BNP-1350), Diflomotecan (BN80915), and Gimatecanetc [6-8]. Tafluposide has been modified from Epipodophyllotoxin, an alkaloid derived from Podophyllum peltatum [9]. Acronycine is an alkaloid derived from Acronychiabaueri that has been modified to S23906-1 [10]. Combretastatin A-4 is an alkaloid derived from Combretum caffrum thathas been converted into anticancer agents such as Combretastatin A-4 phosphate, Combretastatin A-4 diphosphate and Ombrabulin (AVE8062) [11-13]. Terameprocol has been synthesized from 3'-Omethyl-nordihydroguaiaretic acid, a lignin isolated from Larreadivaricatta [14]. Daidzein, an isoflavone found in a variety of plants and herbs such as Pueraria Mirifica, soybeans, and soy products, has been converted to Triphendiol and Genistein $[15,16]$.

Curcumin, one of the active constituents of Curcuma longa L. of the family (Zingiberaceae), has had its structure modified in order to produce biologically active compounds. The chemical modification improved not only the biological activities but also the stability, reducing rotational freedom and minimizing metal-chelation properties [17]. Curcumin analogues demonstrated broad spectrum activities as anticancer, anti-inflammatory, antiHIV, anti-Alzheimer's, antimicrobial, epidermal growth factor receptor (EGFR) inhibitors, and other biological activities as reported in the previous studies [18-28].

Cancer is one of the most dreadful diseases and the leading cause of death today, with nearly 10 million deaths and 19.3 million new cases expected in 2020 [29]. Chemotherapy is the most commonly used method of cancer treatment, but it is always associated with a number of toxic side effects. As a result, we became more reliant on nature, because active constituents of natural origin are assumed to be safe. In some studies, curcumin analogs were also discovered to be an inhibitor of EGFR, one of the primary targets of many anticancer drugs $[18,28]$. Furthermore, many of the cancer cell lines, including breast (MDAMB-231, MCF7, HS 578T, BT-549, T-47D MDA-MB-468, and MDA-MB-468), colon (HCT-116, HT-29, COLO 205, HCT 116, HT29, and SW620), non-small cell lung (A549, NCI-H460, and NCI-H322), renal (786-O), melanoma (SKMEL28), ovarian (SKOV3), and prostate (PC3) cancer cell lines, have been found to express varying levels of EGFR [30-36]. Inspired by their impressive biological activities, we reported herein the molecular docking, antiproliferative potential, and anti-EGFR activity of some of the prepared curcumin analogs $(\mathbf{3} \mathbf{a}, \mathbf{b})$. In silico molecular docking analysis was carried out against EGFR through the Schrodinger's software. As molecular docking is now an integral part of computer-aided drug design (CADD), in which ligands with computed generated 3D structures are positioned into a receptor or biological target in a variety of orientations, conformations, and positions [37], it has numerous uses and applications in drug discovery, including structure-activity studies, lead optimization, and virtual screening to find potential leads [38]. Indeed, molecular docking has been used for more than three decades, and it has resulted in the discovery and development of a large number of new drugs [39].

\section{Methods and Materials}

\subsection{Procurement of Chemicals and Crude Turmeric}

All of the chemicals were obtained from a local vendor. The standard curcumin was obtained from Konark Herbals in India, and the remaining chemicals and reagents were obtained from Merck Chemicals, SD Fine, and CDH. All of the chemicals were either analytical or synthetic grades in nature. The dry turmeric was purchased from a local market and ground into powder.

\subsection{Isolation of Curcumin}

Eighty grams of ground turmeric was refluxed in $200 \mathrm{~mL}$ of dichloromethane for one hour with continuous stirring, followed by filtration under vacuum. The filtrate was 
concentrated under vacuum while the temperature was kept at $50{ }^{\circ} \mathrm{C}$. The reddish yellow oily residue was triturated further with $80 \mathrm{~mL}$ of hexane to yield $2.47 \mathrm{~g}$ of solid residue. The resulting solid residue was dissolved in a small amount of dichloromethane/ methanol (99:1) and loaded into a column packed with silica gel. The column was eluted with the same mobile phase, and curucmin was isolated, collected, and analyzed using thin layer chromatography (TLC) with standard curcumin as a reference. For the isolation of curcumin the conventional method was used and with $80 \mathrm{~g}$ of curcumin powder nearly $244 \mathrm{mg}$ of curcumin was isolated [40].

\subsection{Preparation of Curcumin Analogues}

The isolated curcumin was used as a starting compound for the preparation of curcumin analogues via chemical modification of curcumin according to the methods described $[18,19]$. Figure 1 depicts the chemical modification curcumin and curcumin analogues. An equimolar amount of curcumin (1) $(0.25 \mathrm{mmol} ; 92 \mathrm{mg})$ and 3-chlorophenyl thiourea (2a) $(0.25 \mathrm{mmol} ; 46 \mathrm{mg})$ in glacial acetic acid in $50 \mathrm{~mL}$ round-bottom flask placed in sand bath was heated at $80{ }^{\circ} \mathrm{C}$ with continuous stirring on magnetic stirrer for $10 \mathrm{~h}$ to obtain the compound 3a, while an equimolar amount of curcumin (1) (0.25 mmol; $92 \mathrm{mg})$ and $N$-(4-substitutedphenyl)hydrazine carboxamides $(\mathbf{2 b}, \mathbf{c})(0.25 \mathrm{mmol})$ in glacial acetic acid in $50 \mathrm{~mL}$ round-bottom flask placed in sand bath was heated at $80{ }^{\circ} \mathrm{C}$ with continuous stirring for 8-10 h on magnetic stirrer to obtain the compound $\mathbf{3 b}, \mathbf{c}$. The completion of reaction was monitored throughout by thin layer chromatography using mobile phase, n-hexane: ethylacetate (6:4). The excess of solvent was removed under vacuum distillation. Cooled the reaction mixture to room temperature and poured into the crushed ice to obtain the crude product, filtered, and dried. The crude products were further re-crystallization with ethanol to obtain the compounds, $\mathbf{3 a - c}$. The synthetic route followed is shown in Scheme 1.

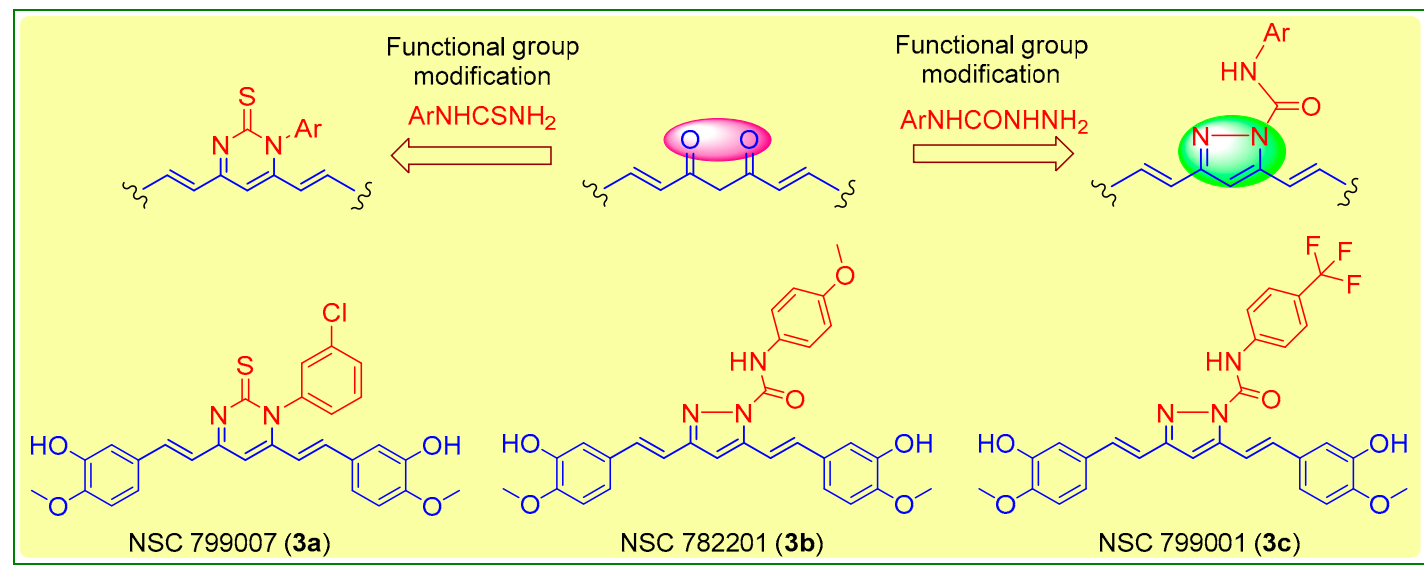

Figure 1. Molecular engineering of curcumin to pyrimidine thione (3a), and pyrazoles $(3 \mathbf{b}, \mathbf{c})$ counterparts.

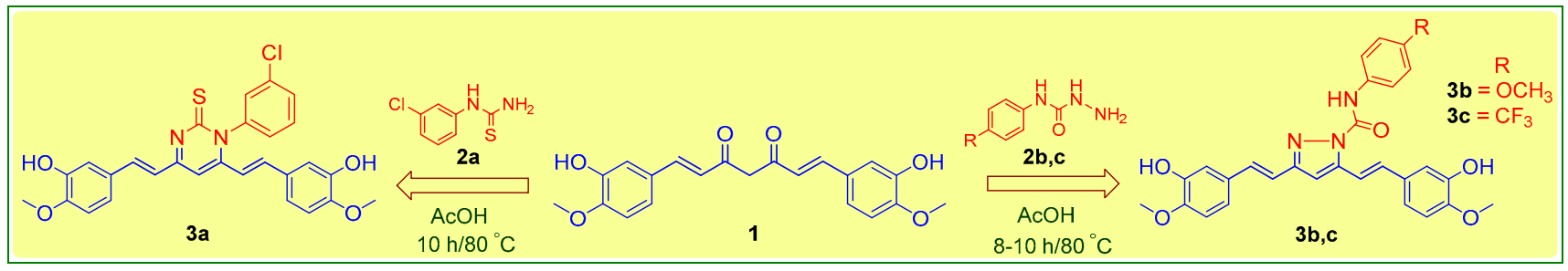

Scheme 1. Molecular engineering for the synthesis of curcumin analogs (3a-c). 


\subsection{Molecular Docking Studies}

The docking was done against epidermal growth factor receptor (EGFR), which is one of the most appealing targets for many anticancer drugs. The protein data bank provided the EGFR (PDB:3W2R) X-ray crystal structure with a resolution of $2.05 \AA$; R-value 0.220 (observed) [41]. The ligands $3 \mathbf{a}-\mathbf{c}$ were saved as mol files, and docking was performed according to the protocol described by Sogabe et al. (2013) [42].

\subsection{Antiproliferative Activity}

The antiproliferative activity of tested compounds was done against five dozen (60) cancer cell lines derived from nine different panels. All of the compounds were evaluated for antiproliferative activity in a single dose at $10 \mu \mathrm{M}$. Growth percent (GP) and percent growth inhibition (\% GI) were used to calculate antiproliferative activity. The tested compounds that demonstrated significant activity were subjected to a five-dose assay $(0.01$ to $100 \mu \mathrm{M})$. The antiproliferative results of five-dose assay were calculated as three dose related parameters like, $50 \%$ growth inhibition $\left(\mathrm{GI}_{50}\right), 50 \%$ lethal concentration $\left(\mathrm{LC}_{50}\right)$, and total growth inhibition TGI [43-47].

Human tumor cell lines were grown in RPMI 1640 medium containing 5\% fetal bovine serum and $2 \mathrm{mM}$ L-glutamine. For a typical screening experiment, cells were inoculated into 96-well microtiter plates in $100 \mu \mathrm{L}$ at plating densities ranging from 5000 to 40,000 cells / well, depending on the doubling time of individual cell lines. Two plates of each cell line were fixed in situ with trichloroacetic acid (TCA) after $24 \mathrm{~h}$ to represent a cell population measurement for each cell line at the time of drug addition (Ti). The experimental drugs were frozen after being solubilized in dimethyl sulfoxide at 400 -fold the desired final maximum test concentration. An aliquot of frozen concentrate was thawed and diluted to twice the desired final maximum test concentration with medium containing $50 \mathrm{\mu g} / \mathrm{mL}$ gentamicin at the time of drug addition. Additional four, 10 -fold, or $\frac{1}{2} \log$ serial dilutions serial dilutions were performed to provide a total of five drug concentrations plus the control. Aliquots of $100 \mu \mathrm{L}$ of these different drug dilutions were added to the appropriate microtiter wells already containing $100 \mu \mathrm{L}$ of medium, resulting in the required final drug concentrations. Following drug addition, the plates are incubated for an additional $48 \mathrm{~h}$ at $37{ }^{\circ} \mathrm{C}, 5 \% \mathrm{CO}_{2}, 95 \%$ air, and $100 \%$ relative humidity. For adherent cells, the assay is terminated by the addition of cold TCA. Cells are fixed in situ by the gentle addition of $50 \mu \mathrm{L}$ of cold $50 \%(w / v)$ TCA (final concentration, 10\% TCA) and incubated for $60 \mathrm{~min}$ at $4{ }^{\circ} \mathrm{C}$. The supernatant is discarded, and the plates are washed five times with tap water and air dried. Sulforhodamine B (SRB) solution $(100 \mu \mathrm{L})$ at $0.4 \%(w / v)$ in $1 \%$ acetic acid was added to each well, and plates were incubated for $10 \mathrm{~min}$ at room temperature. After staining, unbound dye was removed by washing five times with $1 \%$ acetic acid and the plates were air dried. Bound stain was subsequently solubilized with $10 \mathrm{mM}$ trizma base, and the absorbance was read on an automated plate reader at a wavelength of $515 \mathrm{~nm}$. For suspension cells, the methodology was the same except that the assay was terminated by fixing settled cells at the bottom of the wells by gently adding $50 \mu \mathrm{L}$ of $80 \%$ TCA (final concentration, $16 \%$ TCA).

\subsection{Anti-EGFR Activity}

According to the reported method, a spectrophotometric assay for EGFR tyrosine kinase inhibition (absorbance at $\lambda_{\max } 540 \mathrm{~nm}$ ) was performed in human A431 cells, which express higher levels of EGFR protein [48].

\section{Results}

\subsection{Molecular Docking Studies}

EGFR was chosen for docking studies because it is a potential target for many anticancer drugs, including gefitinib, erlotinib, cetuximab, and panitumumab [49]. Curcumin analogues have also been well studied as anti-EGFR agents $[18,28]$. The X-ray crystal structure of EGFR with PDB ID: 3W2R was retrieved from protein data bank [41]. The 
molecular docking score and types of interaction of curcumin analogs (3a-c) are given in Table 1 and binding mode of curcumin analogs are shown in Figure 2. Due to their large structure and high molecular weight, the curcumin analogs fit inside the active site of EGFR and showed various types of interaction including hydrophobic interaction, $\mathrm{H}-\mathrm{bond}$, and $\pi-\pi$ stacking. The ligand 3a displayed only $\pi-\pi$ stacking interaction with the amino acids Asp855, Asp800, and Leu718, while ligand $\mathbf{3 b}$ displayed two H-bond with the residues Leu718 and Asp800 and two $\pi-\pi$ stacking with the residues Leu718 and Asp800 as showed in Figure 3. The ligand $3 \mathbf{c}$ displayed significant interaction within the active site of EGFR having three H-bonds with the residues Met793, Gln791, and Ser720, while three $\pi-\pi$ stacking with the residues Asp855, Asp800, and Leu718 as shown in Figure 4.

Table 1. The molecular docking scores and interaction of ligands 3a-c.

S. No.




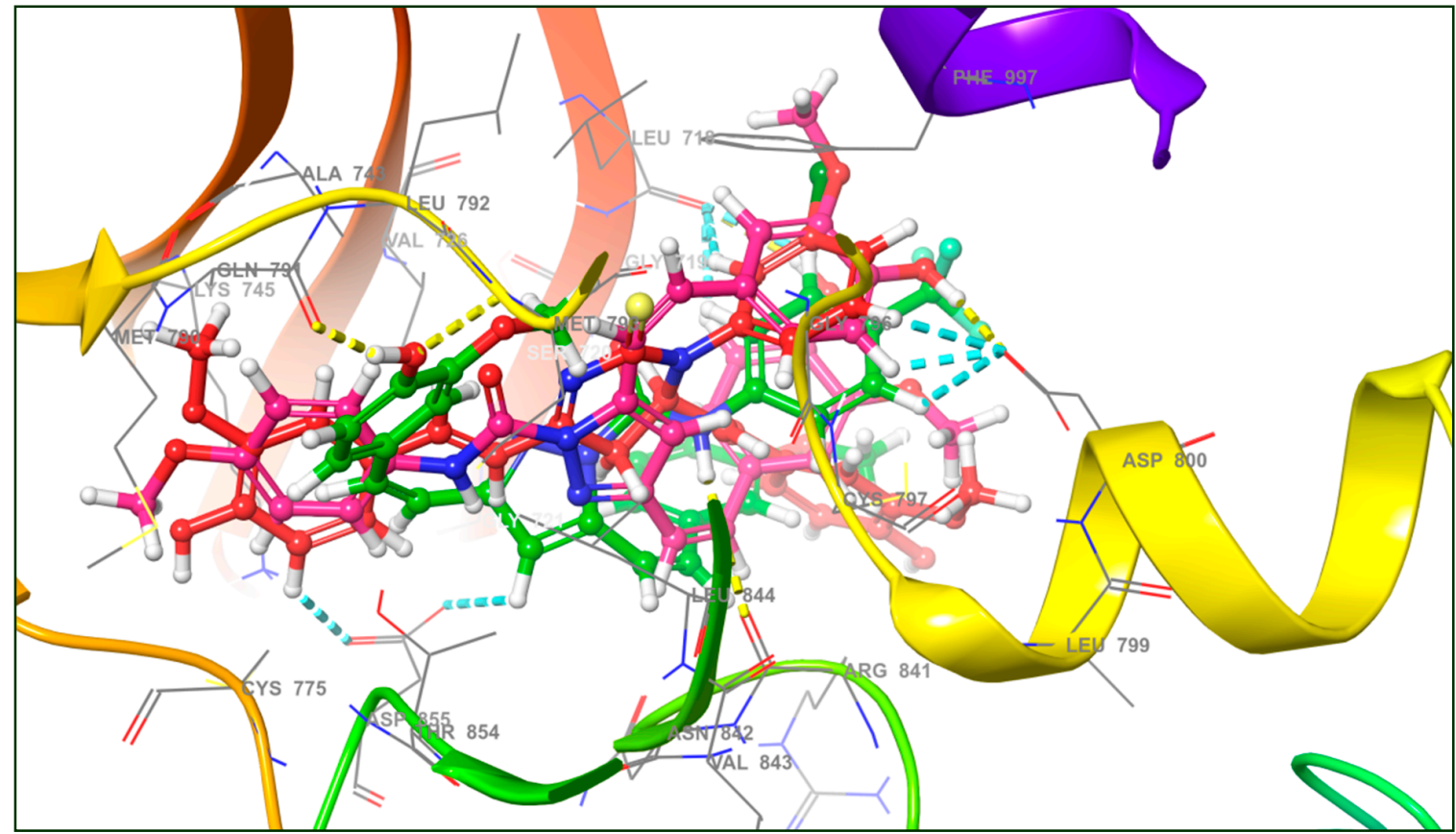

Figure 2. The binding mode of curcumin analogs $(3 \mathbf{a}-\mathbf{c})$ and various types of interactions (yellow dotted line $(-)$ showing H-bond and sky blue dotted line ( - ) showing $\pi-\pi$ stacking) with amino acid residues in docking studies against the active site of EGFR.

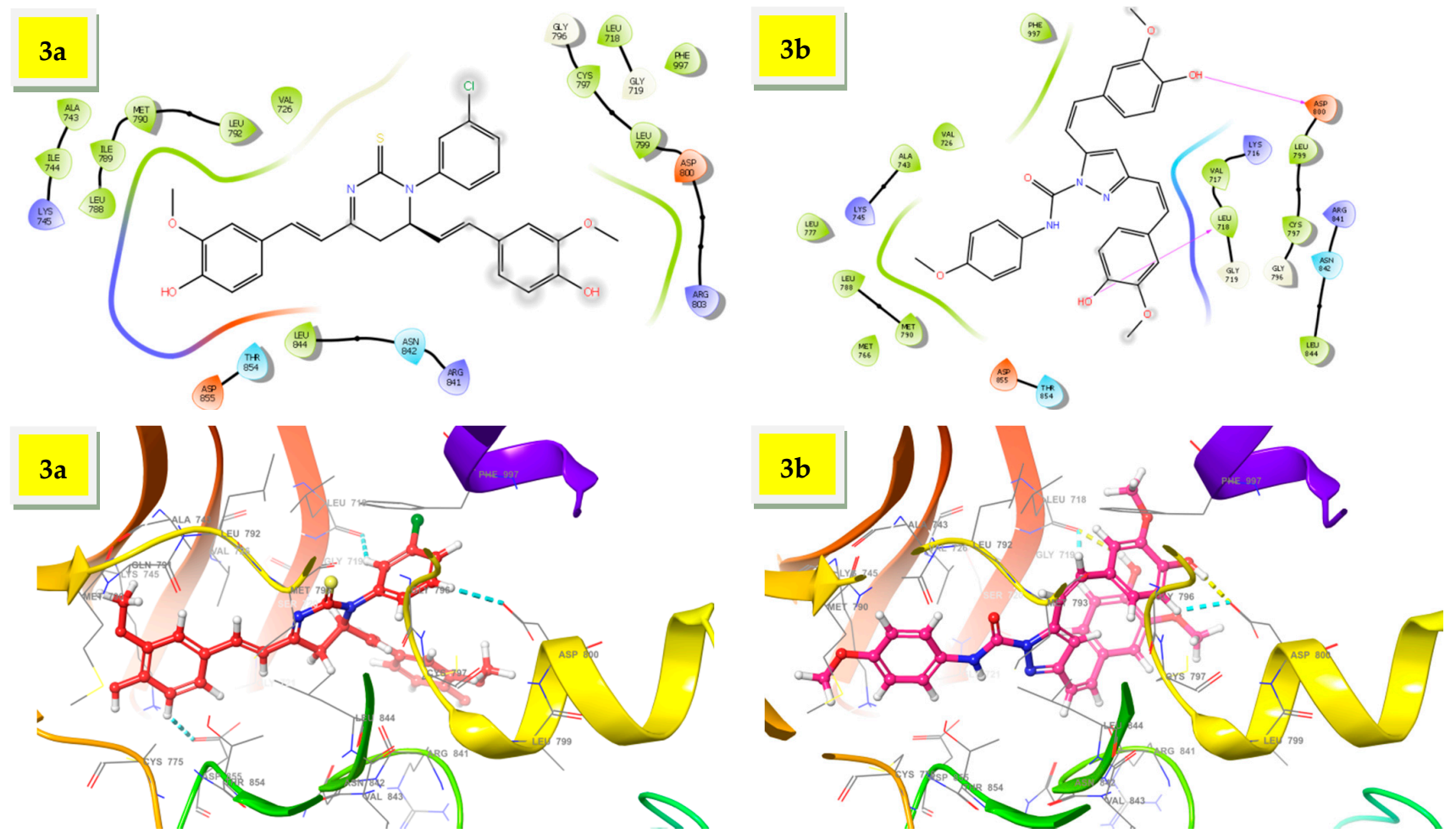

Figure 3. The 3D interaction of ligands, $\mathbf{3 a}, \mathbf{b}$ within the active site of EGFR. 

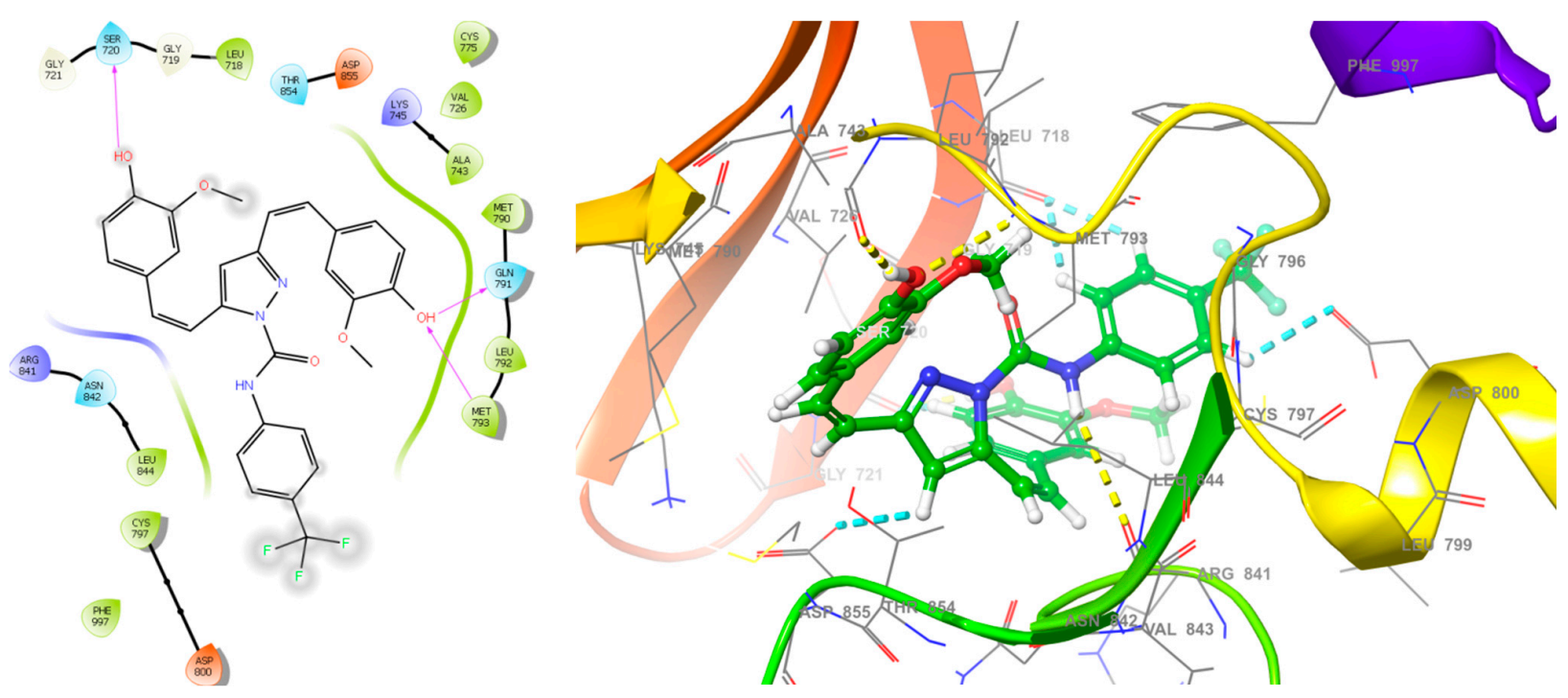

Figure 4. The 2D and 3D interactions of ligand 3c within the active site of EGFR.

\subsection{Antiproliferative Activity}

The ligands $3 \mathbf{a}-\mathbf{c}$ demonstrated significant interactions (docking score: approximately -6.337 to $6.593 \mathrm{kcal} / \mathrm{mol}$ ) in the molecular docking studies and were further tested for antiproliferative activity against five dozen (60) human cancer cell lines derived from nine different panels, including breast, colon, CNS, leukemia, melanoma, non-small cell lung, prostate, and renal cancer cell lines, according to National Cancer Institute (NCI US) protocols [43-47]. In a one-dose assay (Figures S2-S4), compound 3a inhibited growth by more than 50\% in three cancer cell lines-MOLT-4 (\% GI = 53.78), HT29 (\% GI = 53.43), and SR (\% GI = 51.28), while the remaining cell lines (56 cell lines) inhibited growth by less than $50 \%$. Compound $3 \mathbf{b}$ was found to be lethal against two cell lines-NCIH522 and MDA-MB-435, with percent GIs of 113.52 and 136.25, respectively, whereas compound 3c had lethal effect against RPMI-8226 and HCT-116, with percent GIs of 100.21 and 153.67, respectively (shown in red bold font in the Table 2). The compound $3 \mathbf{b}$ was found to be active against 32 cell lines, while the compound $3 \mathrm{c}$ was found to be active against 25 cell lines with \% GIs of $\geq 68 \%$ (shown in green bold font in the Table 2). The compound is supposed to be active if it inhibited the growth of cancer cell lines by $\geq 68$ percent $[50,51]$. Compound $3 \mathbf{b}$ demonstrated $>50 \%$ GIs (and $<68 \%$ GIs) against 13 cell lines, while compound $3 \mathrm{c}$ demonstrated $>50 \%$ GIs against 12 cell lines (shown in blue bold font in the Table 2). Compound $3 \mathbf{b}$ demonstrated $<50 \%$ GIs against 9 cell lines, while compound $3 \mathrm{c}$ demonstrated $<50 \%$ GIs against 17 cell lines (shown in black font in the Table 2). Curcumin was more antiproliferative than compound $\mathbf{3 a}$. Compounds $\mathbf{3 b}$ demonstrated better inhibition than curcumin against 35 of the 56 cell lines in common, while compound $3 c$ displayed better inhibition than curcumin against 27 of the 56 cell lines in common. Figure 5a,b shows a comparison of antiproliferative activity. The results of mean \% GIs on each panel was compared, and it was found that compound $\mathbf{3 b}$ displayed better inhibition than curcumin against seven panels and better inhibition than gefitinib against six cell lines panels, while compound $3 \mathrm{c}$ displayed better inhibition than curcumin against the four cell lines panels and better inhibition than gefitinib against five cell lines panels in one dose assay at $10 \mu \mathrm{M}$ (Table 3). The order of antiproliferative activity of curcumin analogues was found to be $3 \mathbf{b}>3 \mathbf{c}>\mathbf{3 a}$. Compounds $\mathbf{3 b}, \mathbf{c}$ demonstrated promising antiproliferative activity in a single dose assay and thus qualified for further evaluation in a five-dose assay $(0.01,0.10,1,10$, and $100 \mu \mathrm{M})$. 
Table 2. The antiproliferative activity of compounds $\mathbf{3 a}-\mathbf{c}$ in one dose (at $10 \mu \mathrm{M})$ and 5-dose assay (0.01 to $100 \mu \mathrm{M})$ against $60 \mathrm{NCI}$ cancer cell lines.

\begin{tabular}{|c|c|c|c|c|c|c|c|c|c|c|c|c|c|c|c|c|c|}
\hline \multirow{3}{*}{ Panel } & \multirow{3}{*}{ Cell Line } & \multicolumn{6}{|c|}{ GP and $\% \mathrm{GI}$ at $10 \mu \mathrm{M}$} & \multicolumn{5}{|c|}{$3 b$ (NSC 782201) } & \multicolumn{5}{|c|}{$3 b$ (NSC 799011) } \\
\hline & & \multicolumn{2}{|c|}{ 3a (NSC 799007) } & \multicolumn{2}{|c|}{$3 b$ (NSC 782201) } & \multicolumn{2}{|c|}{ 3c (NSC 799011) } & \multirow{2}{*}{$\mathrm{GI}_{50}$} & \multirow{2}{*}{$\begin{array}{l}\text { Sub Panel } \\
\text { MID }^{b}\end{array}$} & \multirow{2}{*}{$\begin{array}{l}\text { Selectivity Ratio } \\
\text { (MID }^{\mathrm{a}} \text { :MID } \\
\text { b) }\end{array}$} & \multirow{2}{*}{ TGI } & \multirow{2}{*}{$\mathrm{LC}_{50}$} & \multirow{2}{*}{$\mathrm{GI}_{50}$} & \multirow{2}{*}{$\begin{array}{l}\text { Sub Panel } \\
\text { MID }^{b}\end{array}$} & \multirow{2}{*}{$\begin{array}{l}\text { Selectivity Ratio } \\
\text { (MID }^{\mathrm{a}}: \text { MID }^{\mathrm{b}} \text { ) }\end{array}$} & \multirow{2}{*}{ TGI } & \multirow{2}{*}{$\mathrm{LC}_{50}$} \\
\hline & & GP & $\% \mathrm{GI}$ & GP & $\% \mathrm{GI}$ & GP & $\%$ GI & & & & & & & & & & \\
\hline \multirow{5}{*}{ Leukemia } & CCRF-CEM & 53.43 & 46.57 & 11.77 & 88.23 & 3.42 & 96.58 & 2.34 & \multirow{5}{*}{2.59} & \multirow{5}{*}{1.89} & 32.7 & $>100$ & 2.63 & \multirow{5}{*}{2.24} & \multirow{5}{*}{1.27} & - & $>100$ \\
\hline & K-562 & 59.23 & 40.77 & 9.25 & 90.75 & 9.45 & 90.55 & 2.87 & & & 16.9 & $>100$ & 2.53 & & & $>100$ & $>100$ \\
\hline & MOLT-4 & 46.22 & 53.78 & 12.24 & 87.76 & 11.09 & 88.91 & 2.87 & & & 13.3 & $>100$ & 2.73 & & & 20.0 & $>100$ \\
\hline & RPMI-8226 & 60.59 & 39.41 & 21.30 & 78.70 & -0.21 & 100.21 & 3.38 & & & 29.2 & $>100$ & 2.09 & & & 6.59 & $>100$ \\
\hline & SR & 48.72 & 51.28 & - & - & 13.21 & 86.79 & 1.52 & & & 12.8 & $>100$ & 1.48 & & & $>100$ & $>100$ \\
\hline \multirow{9}{*}{$\begin{array}{l}\text { Non-Small } \\
\text { Cell Lung } \\
\text { Cancer }\end{array}$} & A549/ATCC & 72.56 & 27.44 & 27.29 & 72.71 & 42.95 & 57.05 & 5.50 & \multirow{9}{*}{5.32} & & 97.9 & $>100$ & 4.11 & & & $>100$ & $>100$ \\
\hline & EKVX & 85.81 & 14.19 & 52.39 & 47.61 & 61.25 & 38.75 & 6.62 & & & 83.7 & $>100$ & 3.22 & & & 38.4 & $>100$ \\
\hline & HOP-62 & 95.06 & 4.94 & 32.75 & 67.25 & 62.87 & 37.13 & 3.65 & & & 42.8 & $>100$ & 3.60 & & & 16.2 & $>100$ \\
\hline & HOP-92 & - & - & 89.07 & 10.93 & - & - & 7.77 & & & $>100$ & $>100$ & 2.35 & & & 6.79 & $>100$ \\
\hline & NCI-H226 & 98.54 & 1.46 & 87.15 & 12.85 & 82.67 & 17.33 & 10.7 & & 0.92 & 78.6 & $>100$ & 7.29 & 3.44 & 0.82 & 27.6 & 91.7 \\
\hline & NCI-H23 & 90.95 & 9.05 & 43.94 & 56.06 & 51.29 & 48.71 & 3.81 & & & 63.8 & $>100$ & 2.13 & & & 6.48 & $>100$ \\
\hline & NCI-H322M & 91.93 & 8.07 & 58.14 & 41.86 & 82.70 & 17.30 & 3.87 & & & 72.5 & $>100$ & 3.25 & & & 12.9 & 49.6 \\
\hline & NCI-H460 & 89.51 & 10.49 & 14.22 & 85.78 & 18.29 & 81.71 & 4.24 & & & 31.4 & $>100$ & 3.22 & & & 9.95 & 50.7 \\
\hline & NCI-H522 & 37.89 & 62.11 & -13.52 & 113.52 & 7.32 & 92.68 & 1.76 & & & 12.7 & $>100$ & 1.78 & & & 4.65 & 40.8 \\
\hline & HCC-2998 & 103.70 & -3.70 & 74.91 & 25.09 & 102.63 & -2.63 & 5.78 & & & $>100$ & $>100$ & 2.04 & & & 3.82 & 71.2 \\
\hline & HCT-116 & 61.88 & 38.12 & 9.13 & 90.87 & -53.67 & 153.67 & 3.22 & & & 42.9 & $>100$ & 1.51 & & & 2.94 & 5.70 \\
\hline $\begin{array}{l}\text { Colon } \\
\text { Cancer }\end{array}$ & HCT-15 & 93.93 & 6.07 & 13.17 & 86.87 & 25.51 & 74.49 & 2.98 & 5.77 & 0.85 & $>100$ & $>100$ & 2.69 & 2.17 & 1.31 & 9.63 & $>100$ \\
\hline & HT29 & 46.57 & 53.43 & 31.26 & 68.74 & 3.50 & 96.50 & 3.52 & & & 11.0 & $>100$ & 2.02 & & & 4.13 & 8.44 \\
\hline & KM12 & 80.33 & 19.67 & 17.89 & 82.11 & 41.38 & 58.62 & 3.19 & & & 20.7 & $>100$ & 2.33 & & & 5.59 & 54.0 \\
\hline & SW-620 & 89.48 & 10.52 & 27.10 & 72.90 & 18.00 & 82.00 & 5.51 & & & 61.6 & $>100$ & 2.56 & & & 5.90 & 33.6 \\
\hline & SF-268 & 83.76 & 16.24 & 41.81 & 58.19 & 37.12 & 62.88 & 3.21 & & & 36.4 & $>100$ & 2.80 & & & 15.4 & $>100$ \\
\hline & SF-295 & 109.94 & -9.94 & 51.62 & 48.38 & 83.42 & 16.58 & 6.25 & & & 53.2 & $>100$ & 2.89 & & & 9.85 & 76.5 \\
\hline CNS Cancer & SF-539 & 93.11 & 6.89 & 6.72 & 93.28 & 29.01 & 70.99 & 2.35 & 383 & 128 & 13.6 & $>100$ & 2.37 & 78 & 100 & 7.45 & 56.0 \\
\hline CNS Cancer & SNB-19 & 84.55 & 15.45 & 38.85 & 61.15 & 48.68 & 51.32 & 5.28 & 3.83 & 1.28 & 86.7 & $>100$ & 3.16 & 2.78 & 1.02 & 11.2 & 36.9 \\
\hline & SNB-75 & 80.78 & 19.22 & 32.61 & 67.39 & 71.80 & 28.20 & 2.35 & & & 16.3 & $>100$ & 2.99 & & & 66.0 & $>100$ \\
\hline & U251 & 59.41 & 40.59 & 26.03 & 73.97 & 9.71 & 90.29 & 3.55 & & & 20.9 & $>100$ & 2.49 & & & 6.86 & $>100$ \\
\hline
\end{tabular}


Table 2. Cont.

\begin{tabular}{|c|c|c|c|c|c|c|c|c|c|c|c|c|c|c|c|c|c|}
\hline \multirow{3}{*}{ Panel } & \multirow{3}{*}{ Cell Line } & \multicolumn{6}{|c|}{ GP and $\% \mathrm{GI}$ at $10 \mu \mathrm{M}$} & \multicolumn{5}{|c|}{$3 b$ (NSC 782201) } & \multicolumn{5}{|c|}{$3 b$ (NSC 799011) } \\
\hline & & \multicolumn{2}{|c|}{ 3a (NSC 799007) } & \multicolumn{2}{|c|}{$3 \mathrm{~b}$ (NSC 782201) } & \multicolumn{2}{|c|}{ 3c (NSC 799011) } & \multirow{2}{*}{$\mathrm{GI}_{50}$} & \multirow{2}{*}{$\begin{array}{l}\text { Sub Panel } \\
\text { MID }^{\mathrm{b}}\end{array}$} & \multirow{2}{*}{$\begin{array}{l}\text { Selectivity Ratio } \\
\left(\text { MID }^{\mathrm{a}}: \text { MID }^{\mathrm{b}} \text { ) }\right.\end{array}$} & \multirow{2}{*}{ TGI } & \multirow{2}{*}{$\mathrm{LC}_{50}$} & \multirow{2}{*}{$\mathrm{GI}_{50}$} & \multirow{2}{*}{$\begin{array}{l}\text { Sub Panel } \\
\text { MID }^{\mathrm{b}}\end{array}$} & \multirow{2}{*}{$\begin{array}{l}\text { Selectivity Ratio } \\
\text { (MID }^{\mathrm{a}}: \text { MID }^{\mathrm{b}} \text { ) }\end{array}$} & \multirow{2}{*}{ TGI } & \multirow{2}{*}{$\mathrm{LC}_{50}$} \\
\hline & & GP & $\%$ GI & GP & $\% \mathrm{GI}$ & GP & $\%$ GI & & & & & & & & & & \\
\hline \multirow{9}{*}{ Melanoma } & LOX IMVI & 78.64 & 21.36 & 27.59 & 72.41 & 9.11 & 90.89 & 3.95 & \multirow{9}{*}{4.59} & \multirow{9}{*}{1.06} & $>100$ & $>100$ & 1.77 & \multirow{9}{*}{2.51} & \multirow{9}{*}{1.31} & 3.71 & - \\
\hline & MALME-3M & 89.46 & 10.54 & 51.86 & 48.14 & 52.27 & 47.73 & 3.66 & & & 38.6 & $>100$ & 2.89 & & & 8.40 & 32.6 \\
\hline & M14 & 94.04 & 5.96 & 4.97 & 95.03 & 29.48 & 70.52 & 3.15 & & & 41.5 & $>100$ & 1.94 & & & 4.08 & 8.58 \\
\hline & MDA-MB-435 & 81.32 & 18.68 & -36.25 & 136.25 & 27.85 & 72.15 & 1.25 & & & 4.68 & $>100$ & 2.35 & & & 7.06 & 33.4 \\
\hline & SK-MEL-2 & 95.15 & 4.85 & 21.38 & 78.62 & 84.47 & 15.53 & 3.23 & & & 18.1 & $>100$ & 3.15 & & & 17.3 & $>100$ \\
\hline & SK-MEL-28 & 92.44 & 7.56 & 34.53 & 65.47 & 57.99 & 42.01 & 3.52 & & & 92.7 & $>100$ & 2.19 & & & 5.90 & 23.9 \\
\hline & SK-MEL-5 & 78.99 & 21.01 & 9.04 & 90.96 & 35.40 & 64.60 & 3.67 & & & 16.1 & $>100$ & 2.27 & & & 6.89 & 28.0 \\
\hline & UACC-257 & 52.91 & 47.09 & 75.22 & 24.78 & 25.01 & 74.99 & 16.7 & & & 64.8 & $>100$ & 4.01 & & & 14.0 & 42.0 \\
\hline & UACC-62 & 82.19 & 17.81 & 25.47 & 74.53 & 31.10 & 68.90 & 2.14 & & & 6.91 & $>100$ & 2.02 & & & 4.76 & 16.7 \\
\hline \multirow{5}{*}{$\begin{array}{l}\text { Ovarian } \\
\text { Cancer }\end{array}$} & IGROV1 & 95.26 & 4.74 & 34.39 & 65.61 & 42.82 & 57.18 & 4.49 & \multirow{5}{*}{6.46} & \multirow{5}{*}{0.76} & 57.0 & $>100$ & 2.77 & & & 11.6 & $>100$ \\
\hline & OVCAR-3 & 91.30 & 8.70 & 13.62 & 86.38 & 13.76 & 86.24 & 2.98 & & & 8.40 & $>100$ & 3.41 & & & 26.7 & $>100$ \\
\hline & OVCAR-4 & 82.55 & 17.45 & 51.51 & 48.49 & 76.71 & 23.29 & 5.72 & & & 90.7 & $>100$ & 3.51 & & & 52.7 & $>100$ \\
\hline & OVCAR-5 & 112.92 & -12.92 & 62.30 & 37.70 & 115.18 & -15.16 & 18.8 & & & 54.1 & $>100$ & 2.16 & 3.24 & 0.88 & 4.97 & 15.2 \\
\hline & OVCAR-8 & 71.43 & 28.57 & 32.79 & 67.21 & 0.43 & 99.57 & 5.32 & & & 59.9 & $>100$ & 3.33 & & & $>100$ & $>100$ \\
\hline & $786-0$ & 94.62 & 5.38 & 32.08 & 67.92 & 20.07 & 79.93 & 4.00 & & & 24.3 & $>100$ & 2.42 & & & 7.52 & $>100$ \\
\hline & A498 & 104.38 & -4.38 & 82.47 & 17.53 & 108.03 & -8.03 & 17.2 & & & 56.4 & $>100$ & 5.81 & & & 2.06 & 57.3 \\
\hline & $\mathrm{ACHN}$ & 95.01 & 4.99 & 26.03 & 77.93 & 65.82 & 34.19 & 4.18 & & & $>100$ & $>100$ & 2.48 & & & 7.08 & $>100$ \\
\hline Renal Cancer & CAKI-1 & 79.04 & 20.96 & 38.06 & 61.94 & 54.99 & 45.01 & 3.60 & 5.84 & 0.84 & $>100$ & $>100$ & 3.02 & 3.06 & 0.93 & 12.7 & 44.1 \\
\hline & RXF 393 & 92.63 & 7.37 & 23.01 & 76.99 & 22.66 & 77.34 & 3.02 & .0. & & 8.87 & $>100$ & 1.98 & 0.00 & & 3.73 & - \\
\hline & SN $12 C$ & 92.61 & 7.39 & 31.45 & 68.55 & 36.92 & 63.08 & 3.05 & & & 33.7 & $>100$ & 2.73 & & & 9.90 & $>100$ \\
\hline & TK-10 & 112.60 & -12.60 & 63.84 & 36.16 & 77.69 & 22.31 & 10.2 & & & 95.3 & $>100$ & 4.13 & & & 14.8 & 59.4 \\
\hline & UO-31 & 71.26 & 28.74 & 22.67 & 77.33 & 42.85 & 57.15 & 1.52 & & & 36.4 & $>100$ & 1.94 & & & - & $>100$ \\
\hline Prostate & PC-3 & 89.21 & 10.79 & 28.43 & 71.57 & 38.03 & 61.97 & 5.11 & 4.55 & 107 & $>100$ & $>100$ & 3.21 & 3.44 & 0.82 & 13.5 & $>100$ \\
\hline Cancer & DU-145 & 80.84 & 19.16 & 27.23 & 72.77 & 47.55 & 52.45 & 3.99 & T.00 & $1.0 \%$ & 24.6 & $>100$ & 3.67 & J.TT & 0.02 & 13.0 & 45.3 \\
\hline
\end{tabular}


Table 2. Cont.

\begin{tabular}{|c|c|c|c|c|c|c|c|c|c|c|c|c|c|c|c|c|c|}
\hline \multirow{3}{*}{ Panel } & \multirow{3}{*}{ Cell Line } & \multicolumn{6}{|c|}{ GP and $\% \mathrm{GI}$ at $10 \mu \mathrm{M}$} & \multicolumn{5}{|c|}{$3 b$ (NSC 782201) } & \multicolumn{5}{|c|}{$3 b$ (NSC 799011) } \\
\hline & & \multicolumn{2}{|c|}{ 3a (NSC 799007) } & \multicolumn{2}{|c|}{$3 b$ (NSC 782201) } & \multicolumn{2}{|c|}{ 3c (NSC 799011) } & \multirow{2}{*}{$\mathrm{GI}_{50}$} & \multirow{2}{*}{$\begin{array}{l}\text { Sub Panel } \\
\text { MID }^{b}\end{array}$} & \multirow{2}{*}{$\begin{array}{l}\text { Selectivity Ratio } \\
\text { (MID }^{\mathrm{a}} \text { :MID }{ }^{\mathrm{b}} \text { ) }\end{array}$} & \multirow{2}{*}{ TGI } & \multirow{2}{*}{$\mathrm{LC}_{50}$} & \multirow{2}{*}{$\mathrm{GI}_{50}$} & \multirow{2}{*}{$\begin{array}{l}\text { Sub Panel } \\
\text { MID }^{b}\end{array}$} & \multirow{2}{*}{$\begin{array}{l}\text { Selectivity Ratio } \\
\left(\text { MID }^{a}: \text { MID }^{b} \text { ) }\right.\end{array}$} & \multirow{2}{*}{ TGI } & \multirow{2}{*}{$\mathrm{LC}_{50}$} \\
\hline & & GP & $\%$ GI & GP & $\%$ GI & GP & $\%$ GI & & & & & & & & & & \\
\hline \multirow{5}{*}{$\begin{array}{l}\text { Breast } \\
\text { Cancer }\end{array}$} & MCF7 & 67.91 & 32.09 & 15.63 & 84.37 & 22.92 & 77.08 & 3.30 & \multirow{5}{*}{4.12} & \multirow{5}{*}{1.19} & 17.0 & $>100$ & 1.97 & \multirow{5}{*}{2.86} & \multirow{5}{*}{0.99} & 10.7 & 72.0 \\
\hline & HS 578T & 84.33 & 15.67 & 28.71 & 71.29 & 51.89 & 48.11 & 6.77 & & & 61.9 & $>100$ & 5.10 & & & 60.6 & $>100$ \\
\hline & BT-549 & 72.52 & 27.48 & 31.37 & 68.63 & 18.97 & 81.03 & 2.49 & & & 29.5 & $>100$ & 1.96 & & & 3.67 & 6.86 \\
\hline & $\mathrm{T}-47 \mathrm{D}$ & 63.82 & 36.18 & 34.83 & 65.17 & 47.40 & 52.60 & 3.68 & & & 25.8 & $>100$ & 2.81 & & & 39.0 & $>100$ \\
\hline & MDA-MB-468 & 83.81 & 16.19 & 34.68 & 65.32 & 43.79 & 56.21 & 3.55 & & & 21.6 & $>100$ & 2.30 & & & 6.25 & $>100$ \\
\hline $\begin{array}{l}\text { Total cell line } \\
\text { and sum of } \\
\text { concentration }\end{array}$ & 60 & & & & & & & 293.96 & & & & & 170.18 & & & & \\
\hline $\mathrm{MID}^{\mathrm{a}}$ & & & & & & & & 4.89 & & & & & 2.84 & & & & \\
\hline
\end{tabular}

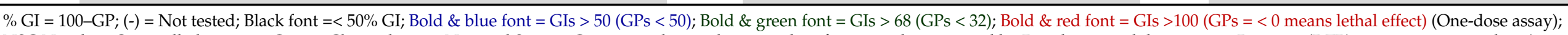

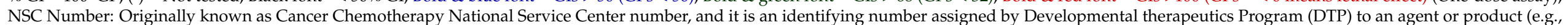

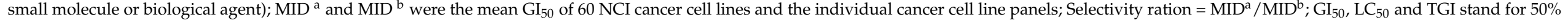
growth inhibition, $50 \%$ lethal concentration, and total growth inhibition respectively. 


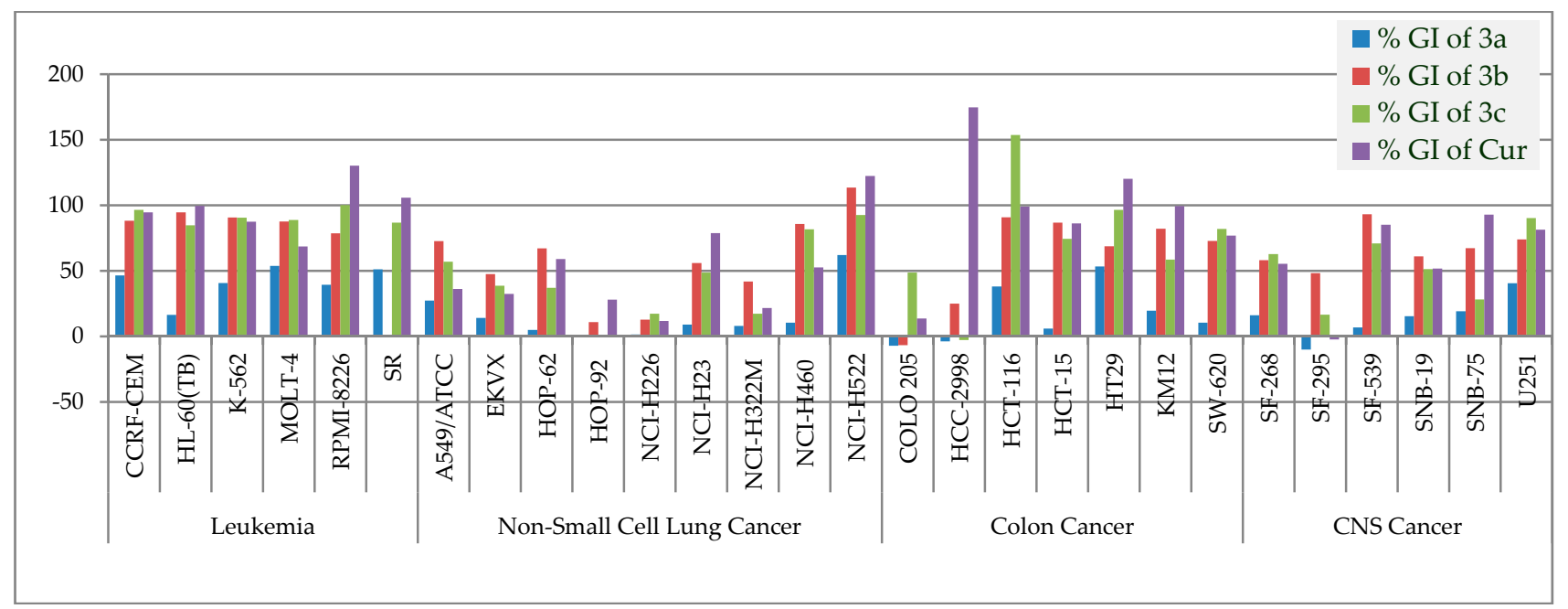

(a)

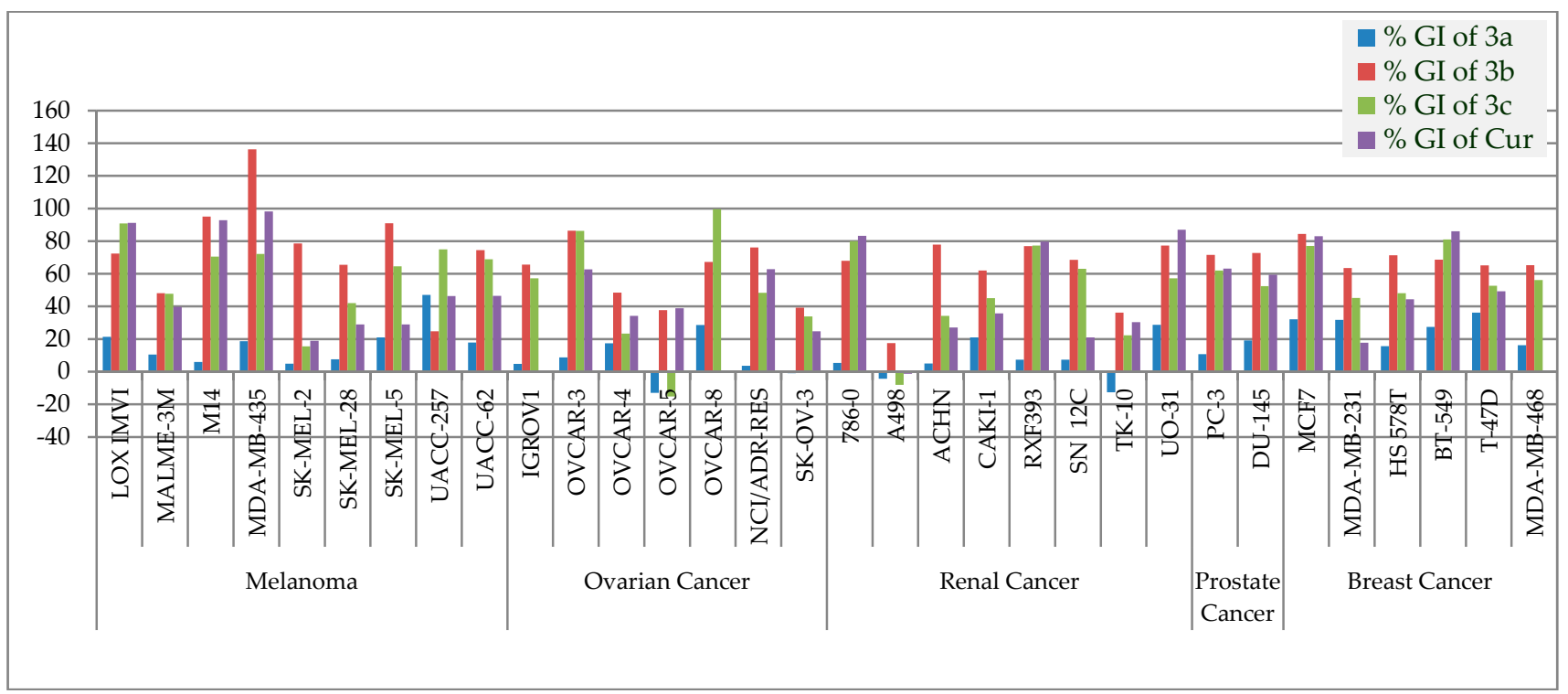

(b)

Figure 5. (a). The \% GIs of curcumin analogs (3a-c) and curcumin (Cur) on leukemia, non-small cell lung, colon, and CNS cancer panels. (b). The \% GIs of curcumin analogs (3a-c) and curcumin (Cur) on melanoma, ovarian, renal, prostate, and breast cancer panels.

Table 3. Mean \% GI of compounds 3a-c, curcumin and gefitinib at $10 \mu \mathrm{M}$.

\begin{tabular}{cccccc}
\hline Panel & $\mathbf{3 a}$ & $\mathbf{3 b}$ & $\mathbf{3 c}$ & Cur $^{*}$ & Gefitinib $^{\text {\# }}$ \\
\hline Leukemia & 41.39 & 88.02 & 91.31 & $\mathbf{9 7 . 7 6}$ & 79.68 \\
Non-Small cell lung cancer & 17.22 & 56.51 & 48.83 & 49.27 & $\mathbf{6 3 . 9 7}$ \\
Colon Cancer & 16.72 & 60 & 73.05 & $\mathbf{9 5 . 7 6}$ & 52.19 \\
CNS Cancer & 14.74 & $\mathbf{6 7 . 0 6}$ & 53.38 & 60.75 & 46.13 \\
Melanoma & 17.21 & $\mathbf{7 6 . 2 4}$ & 60.81 & 54.63 & 44.99 \\
Ovarian Cancer & 7.04 & 60.11 & 47.62 & 44.66 & $\mathbf{6 0 . 9 3}$ \\
Renal Cancer & 7.23 & 60.54 & 46.37 & 45.35 & $\mathbf{7 7 . 8 9}$ \\
Prostate Cancer & 14.98 & $\mathbf{7 2 . 1 7}$ & 57.21 & 61.3 & 59.6 \\
Breast Cancer & 26.56 & $\mathbf{6 9 . 7 1}$ & 60.04 & 56.1 & 52.88 \\
\hline
\end{tabular}

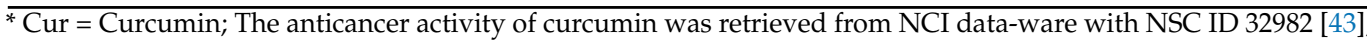
Bold font represents the best result; \# The anticancer activity of gefitinib was retrieved from NCI data-ware with NSC ID 759856 [43]. 
Compounds $3 \mathbf{b}, \mathbf{c}$, which demonstrated promising antiproliferative activity against some of the cancer cell lines in a single dose assay with GIs of $>68$ (GP 32 percent), were chosen for further evaluation in a five-dose assay [50,51]. The antiproliferative activity of compounds $(\mathbf{3 b}, \mathbf{c})$ was represented as a three-dose-related parameter: $\mathrm{GI}_{50}, \mathrm{TGI}$, and $\mathrm{LC}_{50}$. The compound $\mathbf{3 b}$ had $\mathrm{GI}_{50}$ values ranging from 1.25 to $18.8 \mu \mathrm{M}$, TGI values ranging between 6.69 and $>100 \mu \mathrm{M}$ and $\mathrm{LC}_{50}>100 \mu \mathrm{M}$, whereas the compound $3 \mathrm{c}$ had $\mathrm{GI}_{50}$ values ranging from 1.48 to $7.29 \mu \mathrm{M}$, TGI values ranging between 2.06 and $>100 \mu \mathrm{M}$ and $\mathrm{LC}_{50}>100 \mu \mathrm{M}$. Compounds $3 \mathbf{b}, \mathbf{c}$ showed promising inhibitions against SR in leukemia cell lines' panel, with $\mathrm{GI}_{50}$ values of 1.52 and 1.48 , respectively, whereas the compounds $3 \mathbf{b}, \mathbf{c}$ showed promising inhibitions against NCI-H522 in non-small lung cancer cell lines panel with $\mathrm{GI}_{50}$ values of 1.76 and 1.78, respectively. Likewise, compounds $3 \mathbf{b}, \mathbf{c}$ showed promising inhibitions against SF-539 in CNS cell lines' panel, with GI 50 values of 2.35 and 2.37 respectively, whereas the compounds $3 \mathbf{b}, \mathbf{c}$ showed promising inhibitions against UO31 in renal cancer cell lines' panel with $\mathrm{GI}_{50}$ values of 1.52 and 1.94, respectively. Compound $3 \mathbf{b}, \mathbf{c}$ showed promising inhibitions against BT-549 in breast cell lines' panel with GI $_{50}$ values of 2.49 and 1.96 , respectively. Compounds $\mathbf{3 b}$,c showed promising inhibitions, respectively, against HCT-15 and HCT-116 in colon cancer cell lines' panel with GI 50 values of 2.98 and 1.51 respectively, whereas compounds $3 \mathbf{b}$,c showed promising inhibitions, respectively, against MDA-MB-435 and LOXIMVI in melanoma cell lines panel with $\mathrm{GI}_{50}$ values of 1.25 and 1.77 respectively. Compounds $\mathbf{3 b}, \mathbf{c}$ showed promising inhibitions respectively against OVCAR-3 and OVCAR-5 in ovarian cancer cell lines' panel with GI ${ }_{50}$ values of 2.98 and 1.16 , respectively, whereas compounds $3 \mathbf{b}, \mathbf{c}$ showed promising inhibitions, respectively, against DU-145 and PC-3 in prostate cancer cell lines' panel with $\mathrm{GI}_{50}$ values of 3.99 and 3.21 , respectively. Furthermore, the selectivity ration (SR) of compound $3 \mathbf{b}$ ranging between 0.76 and 1.89 , whereas the selectivity ration of compound $3 \mathbf{b}$ ranging between 0.82 and 1.31 indicated their non-selectivity towards the cancer cell line panels. Table 2 summarizes the results of the 5-dose assay. The mean $\mathrm{GI}_{50}$ of each panel was calculated and compared to that of curcumin. Except for the panel colon cancer by compound $\mathbf{3 b}$, both the curcumin analogs $(\mathbf{3 b}, \mathbf{c})$ demonstrated more promising antiproliferative activity with better inhibition than curcumin as shown in Figure 6. The antiproliferative activity of compounds $3 \mathbf{c}$ is shown in Figure 7, while antiproliferative activity of compound $\mathbf{3 b}$ against nine panels of $60 \mathrm{NCI}$ cancer cell lines in terms of GP and Log10 molar concentration are shown in Figure S1 (In Supplementary Materials). In a 5-dose assay, the overall antiproliferative activity was $3 c>3 b>$ curcumin. Curcumin molecular engineering into semi-synthetic curcumin analogues $(3 \mathbf{b}, \mathbf{c})$ resulted in increased antiproliferative activity.

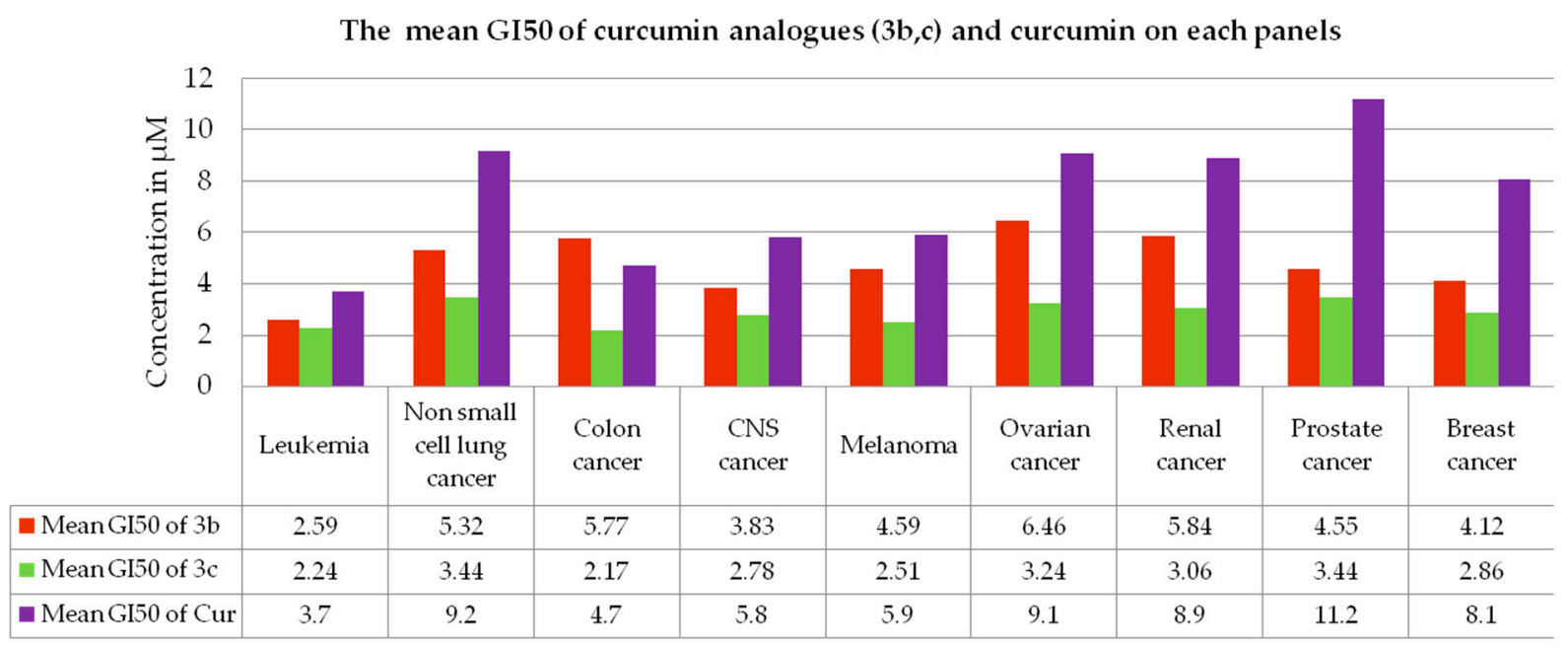

Figure 6. The mean $\mathrm{GI}_{50}$ of curcumin analogs $(3 \mathbf{b}, \mathbf{c})$ and curcumin (Cur) on each panel of cancer cell lines in the fivedose assay. 


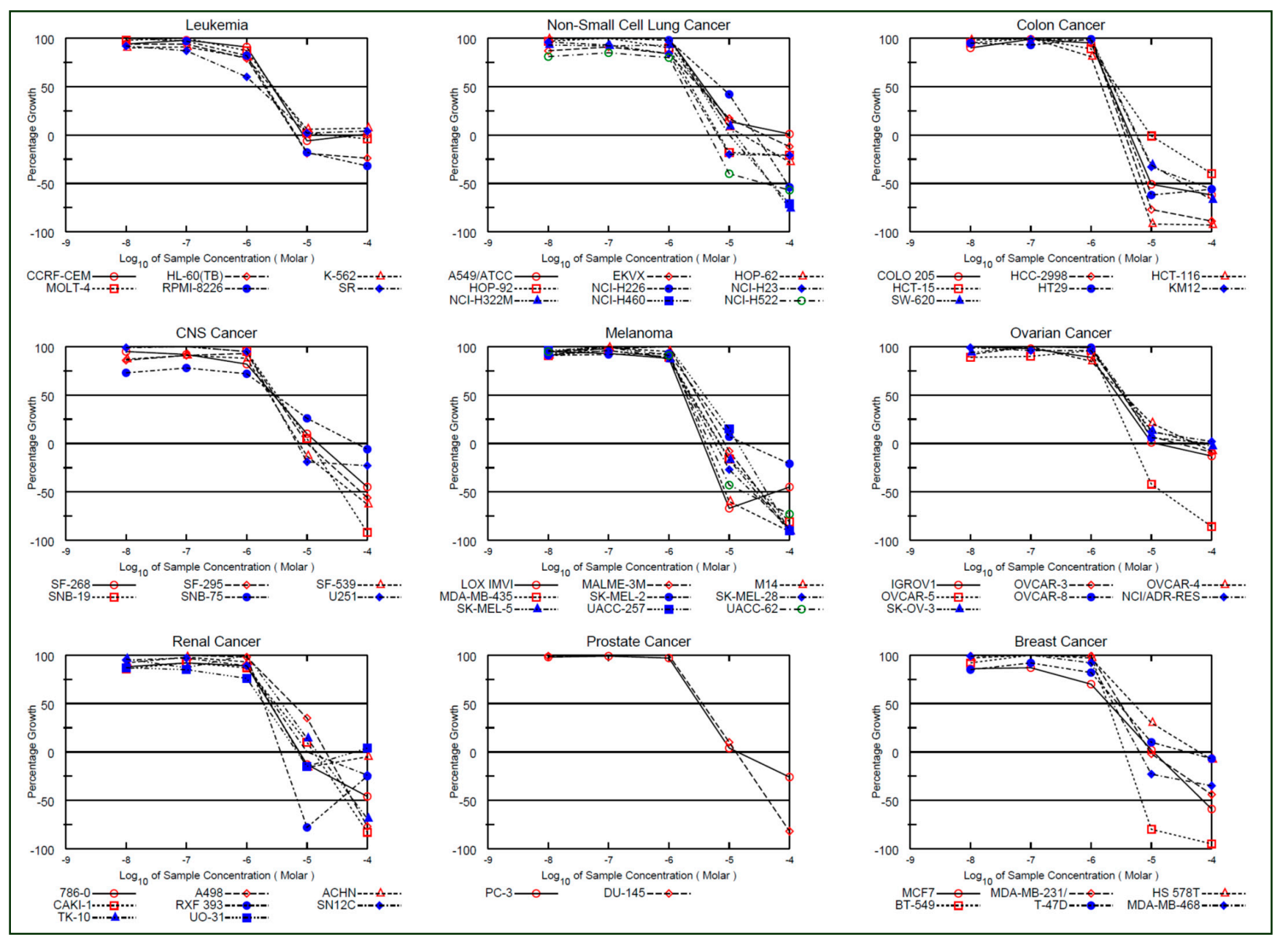

Figure 7. Antiproliferative profile of compound $3 \mathrm{c}$ in terms of GP and $\log _{10}$ molar concentration.

\subsection{Anti-EGFR Activity}

The ligands $(3 \mathbf{b}, \mathbf{c})$ with promising antiproliferative activity and displayed significant interaction within the active site of EGFR in molecular dockingwere then tested for antiEGFR activity in human A431 cells, which express high levels of EGFR protein, using a spectrophotometric assay (absorbance at $\lambda_{\max } 540 \mathrm{~nm}$ ). Both the compounds $(3 \mathrm{~b}, \mathrm{c}$ ) moderately inhibited the EGFR with $\mathrm{IC}_{50}$ values of 3.89 and $4.18 \mu \mathrm{M}$, respectively, while the standard drug, gefitinib inhibited EGFR with $\mathrm{IC}_{50}$ value of $0.017 \mu \mathrm{M}$.

\section{Discussion}

Turmeric has been promoted as a therapeutic herb in the majority of indigenous traditional systems, including Ayurveda, Siddha, Unani, and Chinese medicine [52]. Turmeric's main active ingredient is curcumin, and its stability and bioavailability are the main issues, so it was modified into semi-synthetic analogs to make it more stable and potentiate its bioactivities [17]. Some of the curcumin analogues demonstrated superior antiproliferative activity than curcumin not only in the current study, but also in the previous study, where they demonstrated superior antiproliferative activity in single and five dose assays [18-20].

The molecular docking revealed that compound 3a exhibited two $\pi-\pi$ stacking with the $m$-chlorophenyl moiety with the residues Asp800 and Leu718, as well as one $\pi-\pi$ stacking between one of the methoxyphenyl moiety and the residue Asp855. Compound 3a demonstrated moderate antiproliferative activity with more than 50 percent sensitivity against MOLT-4 (\% GI = 53.78), SR ( $\%$ GI = 51.28), and HT29 (\% GI = 53.43). The compound $\mathbf{3 b}$ displayed two $\mathrm{H}$-bonds with the phenolic functions with the residues Asp800 and 
Leu718, as well as two $\pi-\pi$ stacking one between the methoxyphenyl moiety and the residue Asp855 and another with one of the hydrogen of doubly bonded carbon $(\mathrm{C}=\mathrm{C})$. Compound $\mathbf{3 b}$ exhibited significant interaction within the active site of EGFR in molecular docking studies, and demonstrated better inhibition than curcumin against 35 of the 56 cell lines tested in common in single dose assay at $10 \mu \mathrm{M}$. Compound $3 \mathbf{b}$ inhibited leukemia cancer panel better with $\mathrm{GI}_{50}$ values ranging from 1.52 to $3.38 \mu \mathrm{M}$, but the most promising inhibition with $\mathrm{GI}_{50}$ of $1.25 \mu \mathrm{M}$ was observed against MDA-MB-435, while the least sensitivity was found against OVCAR-5 with $\mathrm{GI}_{50}$ value of $18.8 \mu \mathrm{M}$. Compound $3 \mathrm{c}$ displayed three H-bonds with the phenolic functions with the residues Met793, Ser720, and Gln791, as well as four $\pi-\pi$ stacking three between the $p$-trifluoromethylphenyl moiety and the residues Leu718 and Asp800 and another one with the pyrazoline $\mathrm{CH}$ and residue Asp855. The $p$-trifluoromethyl phenyl function lies within the hydrophobic cavity containing important residues Leu844, Cys797, and Met793. Compound 3c inhibited leukemia cancer panel better with $\mathrm{GI}_{50}$ values ranging from 1.48 to $2.73 \mu \mathrm{M}$, and the most promising inhibition with $\mathrm{GI}_{50}$ of $1.25 \mu \mathrm{M}$ was observed against SR, while the least inhibition was found against NCI-H226 with GI $_{50}$ value of $7.29 \mu \mathrm{M}$. The SI values indicated that both the curcumin analogues $(3 \mathbf{b}, \mathbf{c})$ were found to be non-selective against all the nine panels tested.

The curcumin analogs $(\mathbf{3 a}-\mathbf{c})$ demonstrated promising antiproliferative activity and significant interaction within the active site of EGFR in molecular docking. Previous research has shown that curcumin has anti-EGFR activity [53]. Furthermore, many of the cancer cell lines, including breast, colon, non-small cell lung, renal, melanoma, ovarian, and prostate cancer cell lines, have been found to express varying levels of EGFR; thus, it was worth testing their anti-EGFR activity[30-36]. Compounds (3a-c) were found to be moderately inhibited EGFR. Compound $\mathbf{3 a}$ had a higher docking score than compounds $\mathbf{3} \mathbf{b}, \mathbf{c}$, but it showed moderate antiproliferative and anti-EGFR activities. Compounds $\mathbf{3 b}, \mathbf{c}$, on the other hand, demonstrated significant antiproliferative and anti-EGFR activity, as we observed better interaction within the EGFR active site. Only $\pi-\pi$ stacking interaction was observed with compound $3 \mathbf{a}$, while H-bond and $\pi-\pi$ stacking interactions were observed with compounds $3 \mathbf{b}, \mathbf{c}$.

In most of the cases, the chemical modifications of diketonic function of curcumin into pyrazole and primidinone analogues were found to be promising, whereas chemical modification of diketonic function that resulted in bigenelli-type curcumin compounds were found to be least significant [18-20,54]. Curcumin analogues previously reported in the literature demonstrated cytotoxicity on the CCGF-CEM cell line with $\mathrm{IC}_{50}$ values ranging from 3.13 to $93.40 \mu \mathrm{M}$, whereas compounds $(3 \mathbf{b}, \mathbf{c})$ were found to be much more potent than the reported curcumin analogues [55].

\section{Conclusions}

Three curcumin analogs were prepared and tested for antiproliferative activity $(\mathbf{3 a}-\mathbf{c})$. Compounds $(3 \mathbf{a}-\mathbf{c})$ had docking scores $>-6.337 \mathrm{kcal} / \mathrm{mol}$, indicating efficient binding to the active site of EGFR. Compounds $\mathbf{3 b}, \mathbf{c}$ demonstrated promising antiproliferative activity in both one and five-dose assay. In a one-dose assay, compound $\mathbf{3 b}$ demonstrated the most promising antiproliferative activity with mean \% GI of 66.46, while compound 3c demonstrated the most promising antiproliferative activity in five-dose assay with mean $\mathrm{GI}_{50}$ value of $2.84 \mu \mathrm{M}$, but none of the compounds were found to be selective against any panel in five-dose assay, as indicated by their selectivity ratio values. Except for compound $3 \mathbf{a}$, both compounds $(3 \mathbf{b}, \mathbf{c})$ effectively inhibited the tested cancer cell lines in single and five dose assays. The curcumin analogues moderately inhibited the EGFR, a potential target for anticancer agents. Because the compounds demonstrated promising antiproliferative activity, the current report may lead to an expansion of the anticancer research development program in the future.

Supplementary Materials: The following are available online at https: / www.mdpi.com/article / 10.3390/plants10081559/s1, Figure S1: Antiproliferative profile of compound 3b, Figures S2-S4: Anticancer data of compounds 3a-c in single dose assay. 
Author Contributions: Conceptualization, execution; manuscript writing M.J.A.; synthesis, M.J.A.; molecular docking and drug design, A.A. (Amena Ali); compilation of data and result, A.A. (Amena Ali), M.J.A.; writing-review and editing, M.A.B., A.T. and S.; supervision, M.J.A.; funding acquisition, A.A. (Abuzer Ali). All authors have read and agreed to the published version of the manuscript.

Funding: The research was funded by Taif University Researchers Supporting Project Number (TURSP-2020/124).

Institutional Review Board Statement: Not applicable.

Informed Consent Statement: Not required.

Data Availability Statement: The authors confirm that the data supporting the study's findings are included in the article and its supplementary information.

Acknowledgments: The research was funded by Taif University Researchers Supporting Project Number (TURSP-2020/124). The authors acknowledge the help of National Cancer Institute USA. The authors are also grateful to Schrodinger for providing trial license, and training team.

Conflicts of Interest: The authors declare no conflict of interest.

\section{References}

1. Koehn, F.E.; Carter, G.T. The evolving role of natural products in drug discovery. Nat. Rev. Drug Dis. 2005, 4, 206-220. [CrossRef] [PubMed]

2. Mishra, B.B.; Tiwari, V.K. Natural products: An evolving role in future drug discovery. Eur. J. Med. Chem. 2011, 46, 4769-4807. [CrossRef]

3. Newman, D.J.; Cragg, G.M. Natural products as sources of new drugs from 1981 to 2014. J. Nat. Prod. 2016, 79, 629-661. [CrossRef]

4. Joo, E. Natural Product-Derived Drugs for the Treatment of Inflammatory Bowel Diseases. Intest. Res. 2014, 12, 103-109. [CrossRef]

5. Conlin, A.; de Azambuja, E.; Lago, L.D. Current perspectives of epothilones in breast cancer. Eur. J. Cancer 2008, 44, 341-352.

6. Grossman, S.A.; Carson, K.A.; Phuphanich, S.; Batchelor, T.; Peereboom, D.; Nabors, L.B.; Lesser, G.; Hausheer, F. Phase I and pharmacokinetic study of karenitecin in patients with recurrent malignant gliomas. Neuro-oncology 2008, 10, 608-616. [CrossRef]

7. Butler, M.S. Natural products to drugs: Natural product-derived compounds in clinical trials. Nat. Prod. Rep. 2008, 25, 475 . [CrossRef] [PubMed]

8. Sessa, C.; Cresta, S.; Cerny, T.; Baselga, J.; Caremoli, E.R.; Malossi, A.; Hess, D.; Trigo, J.; Zucchetti, M.; D’Incalci, M.; et al. Concerted escalation of dose and dosing duration in a phase I study of the oral camptothecingimatecan (ST1481) in patients with advanced solid tumors. Ann. Oncol. 2007, 18, 561-568. [CrossRef]

9. Sergent, J.M.; Elgie, A.W.; Williamson, C.J.; Hill, B.T. Ex vivo effects of the dual topoisomerase inhibitor tafluposide (F 11782) on cells isolated from fresh tumor samples taken from patients with cancer. Anti-Cancer. Drug 2003, 14, 467-473. [CrossRef]

10. David-Cordonnier, M.H.; Laine, W.; Lansiaux, A.; Kouach, M.; Briand, G.; Pierré, A.; Hickman, J.A.; Bailly, C. Alkylation of Guanine in DNA by S23906-1, a Novel Potent Antitumor Compound Derived from the Plant Alkaloid Acronycine. Biochemistry 2002, 41, 9911-9920. [CrossRef]

11. Tron, G.C.; Pirali, T.; Sorba, G.; Pagliai, F.; Bussacca, S.; Genzzani, A.A. Medicinal Chemistry of Combretastatin A4: Present and Future Directions. J. Med. Chem. 2006, 49, 3033-3044. [CrossRef] [PubMed]

12. Pettit, G.R.; Lippert, J.W.; Naraynan, V.R.; Varma, R.; Simpson, M.J.; Boyd, M.R.; Rener, G.A.; Bansal, N. Antineoplastic agents 322. synthesis of combretastatin A-4 prodrugs. Anti-Cancer Drug Des. 1995, 10, 299-309.

13. Salmon, H.W.; Siemann, D.W. Effect of the Second-Generation Vascular Disrupting Agent OXi4503 on Tumor Vascularity. Clin. Cancer Res. 2006, 12, 4090-4094. [CrossRef]

14. Grossman, S.A.; Ye, X.; Peereboom, D.; Rosenfeld, M.R.; Mikkelsen, T.; Supko, J.G.; Desideri, S. Phase I study of terameprocol in patients with recurrent high-grade glioma. Neuro. Oncol. 2012, 14, 511-517. [CrossRef] [PubMed]

15. Taylor, C.G.; Feitelson, A.K.; Taylor, D.D. Inhibitory effect of genistein and daidzein on ovarian cancer cell growth. Anticancer Res. 2004, 24, 795-800.

16. Saif, M.W.; Tytler, E.; Lansigan, F.; Brown, D.M. Husband, A.J. Flavonoids, phenoxodiol, and a novel agent, triphendiol, for the treatment of pancreaticobiliary cancers. Expert Opin. Investig. Drugs 2009, 18, 469-479. [CrossRef] [PubMed]

17. Rodrigues, F.C.; Kumar, N.V.A.; Thakur, G. The potency of heterocyclic curcumin analogues: An evidence-based review. Pharmacol. Res. 2021, 166, 105489. [CrossRef]

18. Ahsan, M.J.; Khalilullah, H.; Yasmin, S.; Jadav, S.S.; Govindasamy, J. Synthesis, characterisation, and in vitro anticancer activity of curcumin analogues bearing pyrazole/pyrimidine ring targeting EGFR tyrosine kinase. BioMed Res Int. 2013, 2013, 239354. [CrossRef] [PubMed]

19. Ahsan, M.J.; Choudhary, K.; Jadav, S.S.; Yasmin, S.; Ansari, M.Y.; Sreenivasulsu, R. Synthesis, anticancer activity and molecular docking studies of curcumin analogues bearing pyrazole ring. Med. Chem. Res. 2015, 24, 4166-4180. [CrossRef] 
20. Sharma, R.; Singh, S.; Yasmin, S.; Bhatia, S.; Khalilullah, H.; Ahsan, M.J. Simple, efficient, and improved synthesis of Biginel-li-type compounds of curcumin as anticancer agents. Med. Chem. Res. 2015, 24, 636-644. [CrossRef]

21. Mishra, S.; Karmodiya, K.; Surolia, N.; Surolia, A. Synthesis and exploration of novel curcumin analogues as anti-malarial agents. Bioorg. Med. Chem. 2008, 16, 2894-2902. [CrossRef] [PubMed]

22. Lal, J.; Gupta, S.K.; Thavaselvam, D.; Agrawal, D.D. Design, synthesis, synergistic antimicrobial activity and cytotoxicity of 4-aryl substituted 3,4-dihydropyrimidinones of curcumin. Bioorg. Med. Chem. 2012, 22, 2872-2876. [CrossRef]

23. Sahu, P.K.; Sahu, P.K.; Gupta, S.K.; Thavaselvam, D.; Agarwal, D.D. Synthesis and evaluation of antimicrobial activity of 4H-pyrimido[2,1-b]benzothiazole, pyrazole and benzylidene derivatives of curcumin. Eur. J. Med. Chem. 2012, 54, 366-378. [CrossRef] [PubMed]

24. Saja, K.; Babu, M.S.; Karunagaran, D.; Sudhakaran, P.R. Anti-inflammatory effect of curcumin involves down regulation of MMP-9 in blood mononuclear cells. Int. Immunopharm. 2007, 7, 1659-1667. [CrossRef] [PubMed]

25. Singh, R.K.; Rai, D.; Yadav, D.; Bhargava, A.; Balzarini, J.; DeClercq, E. Synthesis, antibacterial and antiviral properties of curcumin bioconjugates bearing dipeptide, fatty acids and folic acid. Eur. J. Med. Chem. 2010, 45, 1078-1086. [CrossRef] [PubMed]

26. Zhichang, L.; Yinghong, W.; Yuanqin, Z.; Qinxiang, X. Synthesis and antibacterial activities of N-Substituted pyrazole curcumin derivatives. Chin. J. Org. Chem. 2012, 32, 1487-1492.

27. Lee, W.H.; Loo, C.Y.; Bebawy, M.; Luk, F.; Mason, R.S.; Rohanizadeh, R. Curcumin and its Derivatives: Their Application in Neuropharmacology and Neuroscience in the 21st Century. Curr. Neuropharmacol. 2013, 11, 338-378. [CrossRef]

28. Yadav, I.S.; Nandekar, P.P.; Shrivastava, S.; Sanganwar, A.; Choudhry, A.; Agarwal, S.M. Ensemble docking and molecular dynamics identify knoevenagel curcumin derivatives with potent anti-EGFR activity. Gene 2014, 539, 82-90. [CrossRef]

29. Sung, H.; Ferley, J.; Siegel, R.L.; Laversanne, M.; Soerjomartaram, I. Jemal, A.; Bray, F. Global cancer statistics 2020: GLOBOCAN estimates of incidence and mortality worldwide for 36 cancers in 185 countries. CA Cancer J. Clin. 2021, 71, 209-249. [CrossRef]

30. Xu, H.; Yu, Y.; Marciniak, D.; Rishi, A.K.; Sarkar, F.H.; Kucuk, O.; Majumdar, A.P.N. Epidermal growth factor receptor (EGFR)related protein inhibits multiple members of the EGFR family in colon and breast cancer cells. Mol. Can. Ther. 2005, 4, 435-442. [CrossRef]

31. Hoadley, K.A.; Weigman, V.J.; Fan, C.; Sawyer, L.R.; He, X.; Troester, M.A.; Sartor, C.I.; Rieger-House, T.; Bernard, P.S.; Carey, L.A.; et al. EGFR associated expression profiles vary with breast tumor subtype. BMC Genom. 2007, 8, 258. [CrossRef]

32. Rusnak, D.W.; Alligood, K.J.; Mullin, R.J.; Spehar, G.M.; Arenas-Elliott, C.; Martin, A.M.; Degenhardt, Y.; Rudolph, S.K.; Haws, T.F., Jr.; Hudson-Curtis, B.L.; et al. Assessment of epidermal growth factor receptor (EGFR, ErbB1) and HER2 (ErbB2) protein expression levels and response to lapatinib (Tykerb ${ }^{\circledR}$, GW572016) in an expanded panel of human normal and tumour cell lines. Cell Prolif. 2007, 40, 580-594. [CrossRef]

33. Anderson, N.G.; Ahmad, T.; Chan, K.; Dobson, R.; Bundred, N.J. ZD1839 (Iressa), a novel epidermal growth factor receptor (EGFR) tyrosine kinase inhibitor, potently inhibits the growth of EGFR-positive cancer cell lines with or without erbb2 over-expression. Int. J. Can. 2001, 94, 774-782. [CrossRef]

34. Corkey, B.; Crown, J.; Clynes, M.; O'Donovan, N. Epidermal growth factor receptor as a potential therapeutic target in triplenegative breast cancer. Annals Oncol. 2009, 20, 862-867. [CrossRef] [PubMed]

35. Kruwel, T.; Nevoltris, D.; Bode, J.; Dullin, C.; Baty, D.; Chames, P.; Alves, F. In vivo detection of small tumour lesions by multi-pinhole SPECT applying a 99mTc-labelled nanobody targeting the Epidermal Growth Factor Receptor. Sci. Rep. 2016, 6, 21834. [CrossRef] [PubMed]

36. Shaik, N.A.; Al-Kreathy, H.M.; Ajabnoor, G.M.; Verma, P.K.; Banaganapalli, B. Molecular designing, virtual screening and docking study of novel curcumin analogue as mutation (S769L and K846R) selective inhibitor for EGFR. Saudi J. Biol. Sci. 2019, 26, 439-448. [CrossRef] [PubMed]

37. Jakhar, R.; Dangi, M.; Khichi, A.; Chhillar, A.K. Relevance of Molecular Docking Studies in Drug Designing. Curr. Bioinform. 2020, 15, 270-278. [CrossRef]

38. Morris, G.M.; Lim-Wilby, M. Molecular Docking. In Molecular Modeling of Proteins; Humana Press: Totowa, NJ, USA, 2008; pp. 365-382. [CrossRef]

39. Matter, H.; Sotriffer, C. Applications and success stories in virtual screening. In Virtual Screening: Principles, Challenges, and Practical Guidelines; Sotriffer, C., Ed.; Wiley: Weinheim, Germany, 2011; pp. 319-358.

40. Anderson, A.M.; Mitchell, M.S.; Mohan, R.S. Isolation of Curcumin from Turmeric. J. Chem. Edu. 2000, 77, 59-60. [CrossRef]

41. X-ray Crystal Structure of EGFR. Available online: https://www.rcsb.org/structure/3W2R (accessed on 24 May 2021).

42. Sogabe, S.; Kawakita, Y.; Igaki, S.; Iwata, H.; Miki, H.; Cary, D.R.; Takagi, T.; Takagi, S.; Ohta, Y.; Ishikawa, T. Structure-Based Approach for the Discovery of Pyrrolo[3,2-d]pyrimidine-Based EGFR T790M/L858R Mutant Inhibitors. ACS Med. Chem. Lett. 2013, 4, 201-205. [CrossRef]

43. DTP Developmental Therapeutic Programs. Available online: http://dtp.nci.nih.gov (accessed on 9 May 2021).

44. Monks, A.; Scudiero, D.; Skehan, P.; Shoemaker, R.; Paull, K.; Vistica, D.; Hose, C.; Langley, J.; Cronise, P.; Vaigro-Wolff, A.; et al. Feasibility of a highflux anticancer drug screening using a diverse panel of cultured human tumor cell lines. J. Nat. Cancer Inst. 1991, 83, 757-766. [CrossRef]

45. Boyd, M.R.; Paull, K.D. Some practical considerations and applications of the National Cancer Institute in vitro anticancer drug discovery screen. Drug Dev. Res. 1995, 34, 91-109. [CrossRef] 
46. Shoemaker, R.H. The NCI60 human tumour cell line anticancer drug screen. Nat. Rev. Cancer 2006, 6, 813-823. [CrossRef] [PubMed]

47. Grever, M.R.; Schepartz, S.A.; Chabner, B.A. The National Cancer Institute: Cancer drug discovery and development program. Sem. Oncol. 1992, 19, 622-638.

48. Nawaz, F.; Alam, A.; Perwez, A.; Rizvi, A.R.; Naim, M.J.; Siddiqui, N.; Firdaus, J.; Rahman, S.; Jha, M.; Sheikh, A.A. Design, synthesis, molecular docking, and anticancer evaluation of pyrazole linked pyrazoline derivatives with carbothioamide tail as EGFR kinase inhibitors. Anti-Cancer Agent Med. Chem. 2021, 21, 42-60. [CrossRef]

49. Modjtahedi, H.; Essapen, S. Epidermal growth factor receptor inhibitors in cancer treatment: Advances, challenges and opportunities. Anticancer Drugs 2009, 20, 851-855. [CrossRef] [PubMed]

50. Rostom, S.A.F. Synthesis and in vitro antitumor evaluation of some indeno[1,2-c]pyrazol(in)es substituted with sulfonamide, sulfonylurea(-thiourea) pharmacophores, and some derived thiazole ring systems. Bioorg. Med. Chem. 2006, 14, 6475-6485. [CrossRef]

51. Corona, P.; Carta, A.; Loriga, M.; Vitale, G.; Paglietti, G. Synthesis and in-vitro antitumor activity of new quinoxaline derivatives. Eur. J. Med. Chem. 2009, 44, 1579-1591. [CrossRef]

52. Kocaadam, B.; Şanlier, N. Curcumin, an active component of turmeric (Curcuma longa), and Its effects on health. Crit. Rev. Food Sci. Nutr. 2017, 57, 2889-2895. [CrossRef]

53. Starok, M.; Preira, P.; Vayssade, M.; Haupt, K.; Salome, L.; Rossi, C. EGFR Inhibition by Curcumin in Cancer Cells: A Dual Mode of Action. Biomacromolecules 2015, 16, 1634-1642. [CrossRef]

54. Sahu, P.K. Design, structure activity relationship, cytotoxicity and evaluation of antioxidant activity of curcumin derivatives/analogues. Eur. J. Med. Chem. 2016, 121, 510-516. [CrossRef]

55. Seghetti, F.; Di Martino, R.M.C.; Catanzaro, E.; Bisi, A.; Gobbi, S.; Rampa, A.; Canonico, B.; Montanari, M.; Krysko, D.V.; Papa, S.; et al. Curcumin-1,2,3-Triazole Conjugation for Targeting the Cancer Apoptosis Machinery. Molecules 2020, $25,3066$. [CrossRef] [PubMed] 